\title{
A DNA Polymerase with Increased Reactivity for Ribonucleotides and C5-Modified Deoxyribonucleotides
}

\author{
Nadine Staiger and Andreas Marx ${ }^{*[a]}$
}

DNA polymerases find broad applications in molecular biology techniques (e.g., polymerase chain reaction, genome sequencing, or diagnostic methods). ${ }^{[1]}$ Recently, DNA polymerases have increasingly been employed to synthesize highly functionalized DNA and thereby show great promise for future applications ranging from new DNA sequencing technologies ${ }^{[2]}$ to diagnostics, ${ }^{[3]}$ SELEX, ${ }^{[4]}$ and DNA-based nanotechnology. ${ }^{[5]}$ Moreover, it was shown that nucleotide analogues with a simplified sugar backbone such as threosyl or glyceryl surrogates are polymerized by DNA polymerases. ${ }^{[6]}$ Interestingly, these reports show that DNA polymerases from sequence family $B$ are superior to other DNA polymerases in polymerizing highly modified nucleotides. The A485L mutant of Thermococcus species $9^{\circ} \mathrm{N}$ DNA polymerase termed Therminator DNA polymerase (Therminator pol $)^{[7]}$ was widely applied due to its ability to accept several unnatural nucleoside triphosphate analogues as substrates to some extent. However, the observed low efficiency of the enzyme in the processing of several modified substrates often still prevents further applications. Directed evolution shows great promise for improving the performance of DNA polymerases.$^{[8]}$ Here we present the generation of a new Therminator pol variant with broadened substrate scope. The enzyme was identified by a screen of a Therminator pol library that was generated by error-prone PCR. We show that the variant is able to polymerize sugar as well as nucleobase-modified nucleotides to a greater extent than the parental enzyme.

Nucleic acid polymerases are classified either as DNA or RNA polymerases due to their ability to utilize 2'-deoxyribonucleotides (dNTPs) or ribonucleotides (rNTPs) as substrates. DNA and RNA polymerases share a common mechanism for polynucleotide synthesis that leads to the theory of a common ancestor of these enzymes. ${ }^{[9]}$ All DNA polymerases require a primer to initiate DNA synthesis. Usually this is a short RNA primer bound to the canonical template sequence. However, there are a few exceptions like terminal transferases or DNA polymerases that use a protein-primed initiation mechanism. ${ }^{[10]}$ RNA polymerases are dependent on a promoter sequence and start de novo RNA synthesis some nucleotides downstream at the transcription start. ${ }^{[11]}$ Interestingly, widely applied RNA polymerases in in vitro transcription (e.g., T7 RNA polymerase) require processing of purines from the transcription start whereas pyrimidines are poorly processed; this limits the se-

[a] N. Staiger, Prof. Dr. A. Marx

Department of Chemistry and Konstanz Research School Chemical Biology University of Konstanz

Universitätsstrasse 10, 78457 Konstanz (Germany)

Fax: (+49) 7531-88-5140

E-mail:andreas.marx@uni-konstanz.de

Supporting information for this article is available on the WWW under http://dx.doi.org/10.1002/cbic.201000384. quence space available by this method. ${ }^{[11,12]}$ Thus, primer and template-dependent RNA synthesis could overcome this bottleneck. In the last few years, several studies focused on the evolution of DNA polymerases that are able to catalyze promoter-independent synthesis of RNA. ${ }^{[13]}$ Although variants with significantly increased single ribonucleotide incorporation efficiencies were identified, the generated variants mostly abort RNA synthesis after incorporation of a few ribonucleotides. The same holds true for several other tested DNA polymerases (see Figure $\mathrm{S} 1$ in the Supporting Information). Because it has been shown that Therminator pol has some inherent RNA polymerase activity, ${ }^{[14]}$ we became interested in further increasing this ability.

For library construction error-prone $\mathrm{PCR}^{[15]}$ was conducted to introduce random mutations into the Therminator pol gene. Mutants were expressed in 96-well plates by using a synthetic codon-optimized gene whereas the non-codon-optimized approach failed. ${ }^{[16]}$ After heat denaturing of host proteins, the lysates were directly applied in a primer-extension screening assay to identify rNTP-active variants that are able to incorporate multiple ribonucleotides. Because recently described trials using SYBRgreen $I^{[17]}$ failed to detect product formation in solution, screening was performed by employing a chip-based oligonucleotide addressed enzyme assay. ${ }^{[18]}$ Herein, 96 primerextension reactions were conducted in parallel by using an immobilized DNA primer (20 nt)/template $(90 \mathrm{nt})$ complex and rNTPs as substrates. By the partial substitution of UTP with a biotinylated dUTP analogue, biotinylated products were generated in case of any RNA polymerase activity (Figure 1).

Subsequent incubation of the slide surface with a streptavidin-Alexa Fluor 546 conjugate results in a spatially resolved fluorescent signal due to binding to the biotinylated reaction products. Thereby the level of fluorescence correlates with RNA polymerase activity. By using this screening approach 512 rNTP-active variants could be identified that were derived from more than 1700 screened mutants (examples are shown in Figure S2). After further evaluation of these rNTP-active variants by primer extension reactions in solution (data not shown), three mutants (M1, M2, and M3) with enhanced activity for processing of ribonucleotides were obtained. Subsequently, these mutants were purified and thoroughly characterized.

First we conducted a primer-extension reaction employing a 90-nt-long DNA template. Reactions were performed by comparing Thermococcus species $9^{\circ} \mathrm{N}$ DNA polymerase, Therminator pol and the purified mutants. It turned out that variant M3 was able to synthesize the longest RNA products (Figure 2). This variant is able to extend a DNA primer (20 nt)/DNA template $(90 \mathrm{nt})$ complex by consecutive incorporation of more than 50 ribonucleotides, thus generating a $>70$ nt DNA-RNA transcription product. In contrast, $9^{\circ} \mathrm{N}$ DNA polymerase and 

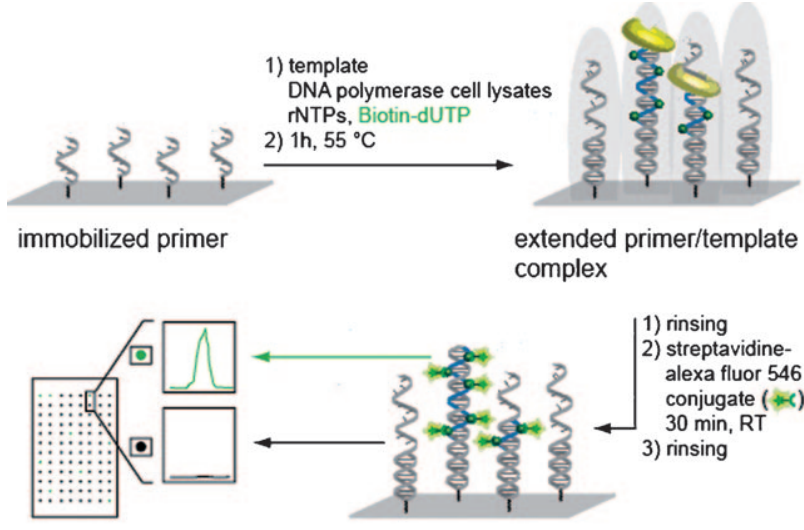

fluorescence read-out

fluorescence-labeled RNA

Figure 1. Principle of chip-based Therminator pol library screening. An immobilized DNA primer strand is shown alone or in complex with the complementary DNA template strand (gray backbone) and the DNA polymerase (yellow). Green stars represent the fluorescent streptavidin-Alexa fluor 546 conjugate bound to the synthesized biotinylated (green dots) RNA strand (blue backbone)
A)

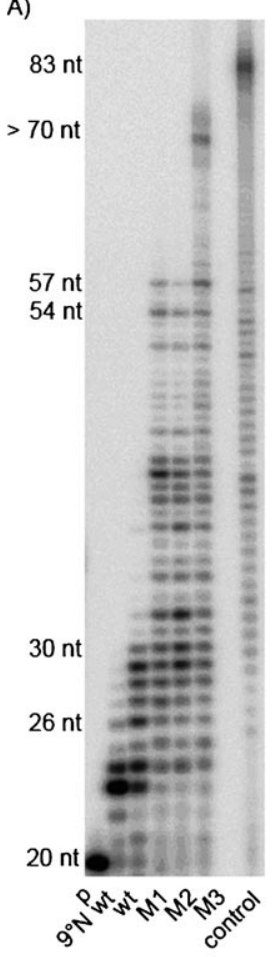

B)

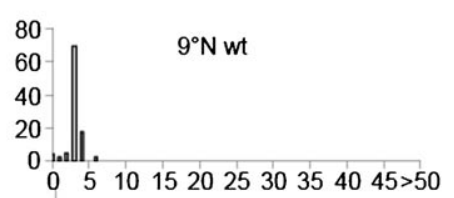

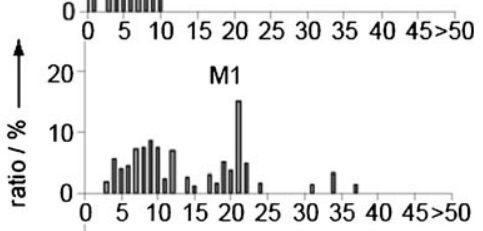
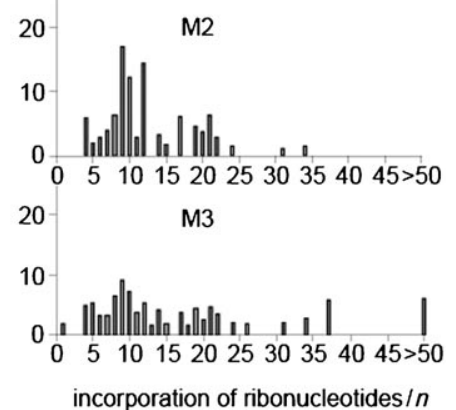

Figure 2. Primer-extension studies with selected Therminator pol variants from chip-based library screening. A) Primer-extension studies in solution. $p$ primer only; $9^{\circ} \mathrm{N} w t: 9^{\circ} \mathrm{N} w t$; Therm wt: Therminator pol wt; M1-3: Therminator pol variants $\mathrm{M} 1-3$; control: primer extension reactions conducted with dNTPs instead of rNTPs. B) Graphical depiction of primer extension reactions from A). Bars indicate the ratio of the extended DNA primer plotted against the number of incorporated ribonucleotides (ratios $>1 \%$ of the total intensity in a single lane were included).

the parental Therminator pol were only able to incorporate up to six and ten ribonucleotides, respectively, under identical conditions. Furthermore, mutants $\mathrm{M} 1$ and $\mathrm{M} 2$ showed a maximum primer extension by conversion of 37 rNTPs or 34 rNTPs,

respectively, which led to DNA-RNA transcription products of 57 and 54 nt in length (Figure 2).

The selected mutants were sequenced and interestingly, they all shared the leucine-to-glutamine mutation at position 408 (L408Q). The two variants with the lower rNTP acceptance (M1 and M2) contained additional amino acid changes (M1: G77S, E276D, A281V, E300K, N568K, Y653C; M2: I171V, K476E, 1488F, 1618F), whereas the most promising mutant (M3) exclusively harbored the $\mathrm{L} 408 \mathrm{Q}$ mutation. Saturation mutagenesis at position $L 408$ and analysis of the resulting mutants did not reveal any variant with improved proficiency for RNA synthesis compared to M3. Based on these results, we selected mutant M3 for further studies.

Next, we investigated whether mutant $M 3$ is able to synthesize RNA transcripts that are functional. Therefore, transcription reactions with an RNA primer (18 nt)/DNA template (53 nt) complex by using purified enzymes were performed to generate an RNA hammerhead ribozyme. ${ }^{[19]}$ After cleavage of DNA templates by DNase I incubation, the purified transcription product was annealed to the ${ }^{32} \mathrm{P}$-labeled RNA substrate and cleavage kinetics were investigated (Figure 3). By using the
A)

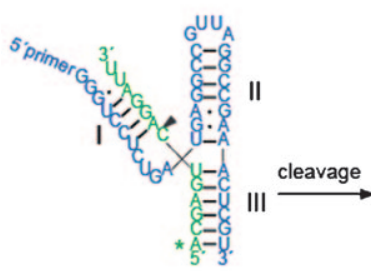

Ribozyme annealed to $5^{\circ}-{ }^{32} \mathrm{P}(*)$-labeled substrate
B)

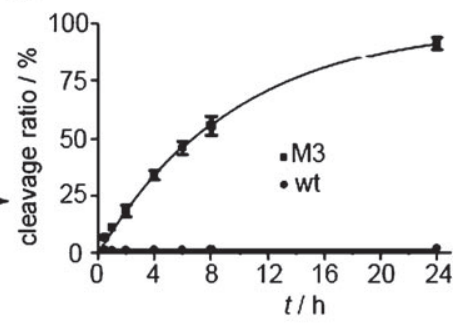

Figure 3. Ribozyme assay. A) RNA ribozyme sequence (blue letters) annealed to the 13 mer substrate (green letters). B) Cleavage activity of hammerhead ribozymes (HHR) transcribed by Therminator pols. Cleavage ratios were obtained after analyzing the reaction products from ribozyme assays performed with HHRs transcribed by Therminator pol variant M3 (घ) or Therminator pol wt (•).

RNA obtained by primer extension with mutant M3 more than $90 \%$ substrate conversion could be detected; this shows that the obtained RNA is indeed functional. This contrasts the results obtained with the parental enzyme in which no significant activity was detected (Figure 3B).

In further experiments we tested the ability of mutant M3 to catalyze the promoter-independent synthesis of tRNA transcripts. Unlike the parental Therminator pol, mutant M3 is proficient in synthesizing full-length tRNA transcripts by the consecutive incorporation of 58 ribonucleotides subsequent to an $18 \mathrm{nt}$ RNA primer (Figure 4). This feature could progress the enzymatic synthesis of tRNA sequences by starting with pyrimidines that are poorly processed in in vitro transcription by using widely applied promoter-dependent RNA polymerases. ${ }^{[11,12]}$ Additionally, by using 5'-modified RNA primers, tRNA sequences with 5'modifications such as fluorescent labels could be achieved, enabling the monitoring of labeled tRNAs. ${ }^{[20]}$

To investigate the influence of the amino acid substitution L408Q on nucleotide incorporation efficiencies as well as 


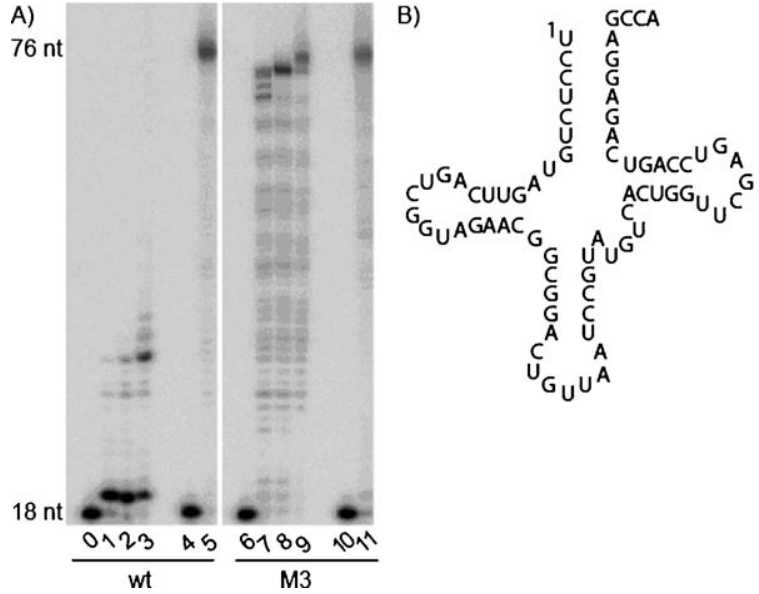

Figure 4. Synthesis of tRNA by using Therminator DNA polymerases. A) Primer-extension studies in solution. Lanes $0,4,6,10$ : primer only; lanes 1, 2, 3: Therminator pol wt applying different reaction times of 1, 3, and $22 \mathrm{~h}$, respectively; lane 5: positive control, reactions with Therminator pol wt and dNTPs; lanes 7, 8, 9: DNA polymerase mutant M3 at different reaction times of 1,3 , and $22 \mathrm{~h}$, respectively; lane 11: positive control, reactions with mutant $\mathrm{M} 3$ and dNTPs; B) Depiction of structure and transcribed RNA sequence of $E$. coli tRNA ${ }^{A s n}$; modified sequence from transfer RNA database (tdbD00001950), University of Leipzig. ${ }^{[21]}$

enzyme selectivity, steady-state kinetic analysis ${ }^{[22]}$ was conducted under single completed hit conditions with the parental enzyme and the variant M3. For this, a gel-based single-nucleotide insertion assay was employed with an RNA primer (18 nt)/ DNA template $(28 \mathrm{nt})$ complex that directs for the canonical (dG) or noncanonical (dA) insertion of cytidines subsequent to the primer end. Overall, single-nucleotide incorporation kinetic analysis showed that variant M3 incorporates $\mathrm{rCTP}$ with a 750fold enhanced efficiency opposite dG compared to the parental enzyme (Table S1). Interestingly, this variant converts dCTP fivefold more efficient than the parental enzyme. Thereby, only a 13-fold drop (efficiency of dCTP processing opposite matched template $d G$ versus mismatched $d A$ by wt in comparison to M3) in mismatch discrimination with dCTP as a substrate was determined. However, variant M3 exhibits an 840fold higher efficiency for processing of rCTP opposite a matched $(d G)$ in comparison to a mismatched (dA) DNA template.

Numerous nucleobase-modified nucleotides are substrates for DNA polymerases and are employed in a wide range of biotechnological applications ranging from SELEX to new sequencing technologies and DNA-based nanotechnology. ${ }^{[2-5]}$ Often the respective DNA is modified by employing C5-modified deoxyuridine. Although known DNA polymerases have some ability to process these analogues, improved activities would be desirable for many applications. Thus, we further investigated the ability of $\mathrm{M} 3$ to incorporate multiple $\mathrm{C} 5$-modified deoxyribonucleotides in a growing DNA strand. Here we used a recently developed nucleotide that bears a bulky nitroxyl radical at position C5. $^{[23]}$ Multiple incorporation of stable radicals would allow high-density radical modification of DNA and generate a material that is interesting for development of organic radical batteries. ${ }^{[24]}$ Primer extension reactions were performed by applying a DNA primer (23 nt)/DNA template (69 nt) complex that codes for the consecutive incorporation of 46 C5-modified deoxyribonucleotides. When employing dNTPs, both Therminator DNA polymerases resulted in fulllength product (Figure $5 \mathrm{~A}$, lanes 2 and 8); meanwhile only variant M3 proficiently incorporated 46 consecutive C5-modified deoxyribonucleotides (Figure 5 A, lanes 3-5 and 9-11).
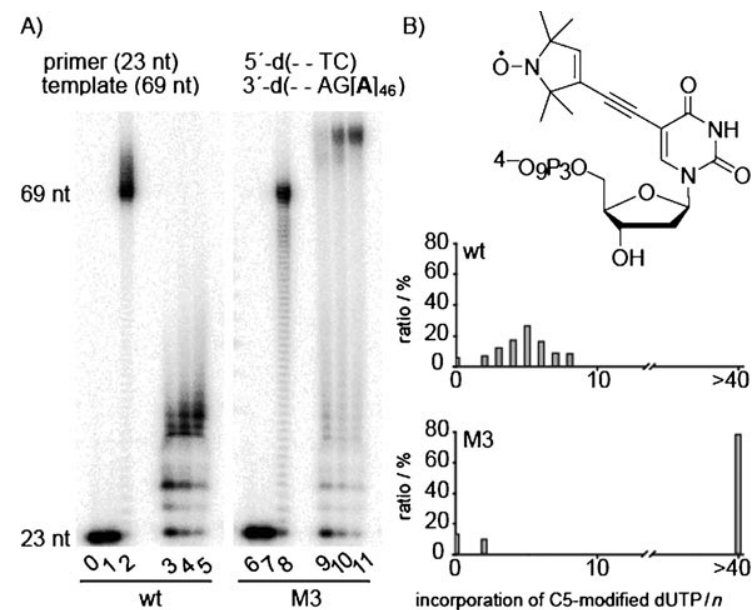

Figure 5. Incorporation of C5-modified deoxyribonucleotides by Therminator DNA polymerases. A) Primer extension reactions in solution employing Therminator pol (wt) or Therminator M3 (M3). Partial primer and template sequences are depicted above the corresponding PAGE analysis. Lanes 0,6 : primer only; lanes 1, 7: negative control, reactions without TTP; lanes 2, 8: positive control, reactions with natural TTP; lanes 3-5 and 9-11: reactions with C5-modified deoxyuridine applying different reaction times, lane 3, 9: $2 \mathrm{~min}$; lane 4, 10: $5 \mathrm{~min}$; lane 5, 11: $10 \mathrm{~min}$. B) Graphical depiction of reactions from (A) lanes 5, 11. Bars indicate the ratio of the extended DNA primer plotted against the number of incorporated ribonucleotides (ratios $>5 \%$ of the total intensity in a single lane were included). For experimental details see the Supporting Information. Inset: Structure of C5-modified deoxyuridine.

Our study revealed that the single amino acid substitution Leu408GIn (M3) in the active site of the B family Therminator pol leads to an increase in DNA-directed RNA synthesis (Figure 2 and 4). The identified residue Leu408 is located in motif $A$ of the polymerase domain. Sequence alignment shows that this Leu408 residue is in the analogous position to Leu415 (RB69) and Ile614 (Taq pol I; Figure S3). These two residues are found to be involved in substrate discrimination in $B$ family RB69 and A family Taq poll, respectively. The substituted amino acid Leu408 in Therminator pol is furthermore located adjacent to a tyrosine (Tyr409) that is in the analogous position to Y416 from RB69 DNA polymerase and E615 from Taq DNA polymerase, which have been shown to act as "steric gates" preventing ribonucleotide incorporation by a steric clash with the $2^{\prime}-\mathrm{OH}$ group of the sugar moiety of an incoming ribonucleotide. ${ }^{[13 c, f]}$ Due to this, it can be assumed that the altered interaction of amino acid 408 with the "steric gate" residue Tyr409 is responsible for the increased ribonucleotide acceptance, by impeding the "steric clash" with the $2^{\prime}-\mathrm{OH}$ group of an incoming ribonucleotide. Furthermore, the substitution of Leu408 with Gln resulted in an improved utilization of C5modified deoxyribonucleotides (Figure 5, lanes 9-11). Presuma- 
bly, the altered active-site geometry of variant M3 enables better accommodation of the bulky nucleotide analogue as well as of the primer extension multiple C5-modified dsDNA product, thus enabling efficient incorporation of $\mathrm{C} 5$-modified deoxyribonucleotides.

In summary, we have generated a Therminator DNA polymerase variant that is capable of promoter-independent synthesis of tRNA as well as synthesizing functional RNA with significantly increased efficiency compared to the parental enzyme. The enzyme variant was identified by screening a small Therminator pol library by using a chip-based oligonucleotide-addressed enzyme assay. Our results show that the highly conserved Leu408 residue, which is located in the conserved motif $A$ of the polymerase domain, is involved in substrate discrimination. We also demonstrate that the identified enzyme variant is capable of synthesizing highly modified DNA by using C5-modifed deoxyribonucleotides as substrates. Moreover, a comparison to the meanwhile commercially available Therminator variants reveals that $\mathrm{M} 3$ is better at processing ribonucleotides and C5-modifed deoxyribonucleotides (Figures S4 and S5). Because this kind of nucleotide analogue is employed in biotechnological applications such as SELEX and next-generation gene sequencing, our findings should spur progress along these lines.

\section{Acknowledgements}

We gratefully acknowledge funding by the DFG. Providing C5modified deoxyribonucleotide by Samra Obeid is kindly acknowledged.

Keywords: DNA polymerase $\cdot$ enzyme engineering $\cdot$ modified nucleotide $\cdot$ ribozyme $\cdot$ RNA

[1] J. Sambrook, D. W. Russell, Molecular Cloning: A Laboratory Manual, Cold Spring Harbor Laboratory Press, New York, 2001.

[2] a) D. R. Bentley, S. Balasubramanian, H. P. Swerdlow, G. P. Smith, J. Milton, C. G. Brown, K. P. Hall, D. J. Evers, C. L. Barnes, H. R. Bignell et al., Nature 2008, 456, 53; b) T. D. Harris et al., Science 2008, 320, 106.

[3] Recent examples: a) V. Raindlová, R. Pohl, M. Sanda, M. Hocek, Angew. Chem. 2010, 122, 1082; Angew. Chem. Int. Ed. 2010, 49, 1064; b) P. M. E. Gramlich, C. T. Wirges, A. Manetto, T. Carell, Angew. Chem. 2008, 120, 8478; Angew. Chem. Int. Ed. 2008, 47, 8350; c) H. Cahová, L. Havran, P. Brázdilová, H. Pivoňková, R. Pohl, M. Fojta, M. Hocek, Angew. Chem. 2008, 120, 2089; Angew. Chem. Int. Ed. 2008, 47, 2059; d) D. M. Hammond, A. Manetto, J. Gierlich, V. A. Azov, P. M. Gramlich, G. A. Burley, M. Maul, T. Carell, Angew. Chem. 2007, 119, 4262; Angew. Chem. Int. Ed. 2008, 47, 4184; e) H. Weizman, Y. Tor, J. Am. Chem. Soc. 2002, 124, 1568

[4] a) M. Famulok, J. S. Hartig, G. Mayer, Chem. Rev. 2007, 107, 3715; b) M. Hollenstein, C. Hipolito, C. Lam, D. Dietrich, D. M. Perrin, Angew. Chem. 2008, 120, 4418; Angew. Chem. Int. Ed. 2008, 47, 4346; c) M. Hollenstein C. J. Hipolito, C. H. Lam, D. M. Perrin, Nucleic Acids Res. 2009, 37, 1638; d) C. G. Peng, M. J. Damha, J. Am. Chem. Soc. 2007, 129, 5310; e) S Jäger, M. Famulok, Angew. Chem. 2004, 116, 3399; Angew. Chem. Int. Ed. 2004, 43, 3337; f) S. Jäger, G. Rasched, H. Kornreich-Leshem, M. Engeser, O. Thum, M. Famulok, J. Am. Chem. Soc. 2005, 127, 15071.

[5] a) W. M. Shih, J. D. Quispe, G. F. Joyce, Nature 2004, 427, 618; b) C. Lin, X. Wang, Y. Liu, N. C. Seeman, H. Yan, J. Am. Chem. Soc. 2007, 129, 14475 ; c) S. Keller, J. Wang, M. Chandra, R. Berger, A. Marx, J. Am. Chem. Soc. 2008, 130, 13188; d) M. Safak, F. E. Alemdaroglu, Y. Li, E. Ergen, A Herrmann, Adv. Mater. 2007, 19, 1499; e) F. E. Alemdaroglu, W. Zhuang,
L. Zöphel, J. Wang, R. Berger, J. P. Rabe, A. Herrmann, Nano Lett. 2009 9, 3658; f) F. E. Alemdaroglu, J. Wang, M. Börsch, R. Berger, A. Herrmann, Angew. Chem. 2008, 120, 988; Angew. Chem. Int. Ed. 2008, 47, 974; A. Baccaro, A. Marx, Chem. Eur. J. 2010, 16, 218.

[6] a) J. K. Ichida, K. Zou, A. Horhota, B. Yu, L. W. McLaughlin, J. W. Szostak J. Am. Chem. Soc. 2005, 127, 2802; b) A. Horhota, K. Zou, J. K. Ichida, B. Yu, L. W. McLaughlin, J. W. Szostak, J. C. Chaput, J. Am. Chem. Soc. 2005 127, 7427; c) J. J. Chen, C.-H. Tsai, X. Cai, A. T. Horhota, L. W. McLaughlin J. W. Szostak, PLoS One 2009, 4, e4949.

[7] A. F. Gardner, W. E. Jack, Nucleic Acids Res. 2002, 30, 605.

[8] Reviews: a) S. Brakmann, Cell. Mol. Life Sci. 2005, 62, 2634; b) R. C. Holmberg, A. A. Henry, F. E. Romesberg, Biomol. Eng. 2005, 22, 39; c) D. Loakes, P. Holliger, Chem. Commun. 2009, 4619.

[9] a) M. Delarue, O. Poch, N. Tordo, D. Moras, P. Argos, Protein Eng. 1990 3,461 ; b) H. Pelletier, M. R. Sawaya, A. Kumar, S. H. Wilson, J. Kraut, Science 1994, 264, 1891; c) T. A. Steitz, S. J. Smerdon, J. Jäger, C. M. Joyce, Science 1994, 266, 2022; d) G. Gao, M. Orlova, M. M. Georgiadis, W. A. Hendrickson, S. P. Goff, Proc. Natl. Acad. Sci. USA 1997, 94, 407.

[10] a) K. Ramadan, I. V. Shevelev, G. Maga, U. Hübscher, J. Mol. Biol. 2004, 339, 395; b) U. Hübscher, S. Spadari, G. Villani, DNA Polymerases: Discovery, Characterization and Functions in Cellular DNA Transactions, World Scientific Publishing, Singapore, 2010; c) S. Kamtekar, A. J. Berman, J. Wang, J. M. Lázaro, M. de Vega, L. Blanco, M. Salas, T. A. Steitz, Mol. Cell 2004, 16, 609

[11] R. Losick, M. Chamberlin, RNA Polymerase, Cold Spring Harbor Laboratory Press, New York, 1976.

[12] For example: a) S. R. Price, N. Ito, C. Oubridge, J. M. Avis, K. Nagai, J. Mol. Biol. 1995, 249, 398; b) I. Kuzmine, P. A. Gottlieb, C. T. Martin, J. Biol. Chem. 2003, 278, 2819; c) T. A. Steitz, EMBO J. 2006, 25, 3458.

[13] a) G. Xia, L. Chen, T. Sera, M. Fa, P. G. Schultz, F. E. Romesberg, Proc. Natl. Acad. Sci. USA 2002, 99, 6597; b) M. Fa, A. Radeghieri, A. A. Henry, F. E. Romesberg, J. Am. Chem. Soc. 2004, 126, 1748; c) P. H. Patel, L. A. Loeb, J. Biol. Chem. 2000, 275, 40266; d) M. Astatke, K. Ng, N. D. F. Grindley, C. M. Joyce, Proc. Natl. Acad. Sci. USA 1998, 95, 3402; e) A. F. Gardner, W. E. Jack, Nucleic Acids Res. 1999, 27, 2545; f) G. Yang, M. Franklin, J. Li, T.-C. Lin, W. Konigsberg, Biochemistry 2002, 41, 10256; g) A. F. Gardner, C. M. Joyce, W. E. Jack, J. Biol. Chem. 2004, 279, 11834; h) A. Bonnin, J. M. Lázaro, L. Blanco, M. Salas, J. Mol. Biol. 1999, 290, 241; i) J. L. Ong, D. Loakes, S. Jaroslawski, K. Too, P. Holliger, J. Mol. Biol. 2006, 361, 537.

[14] E. O. McCullum, J. C. Chaput, Chem. Commun. 2009, 2938.

[15] R. C. Cadwell, G. F. Joyce, Genome Res. 1992, 2, 28.

[16] M. Welch, S. Govindarajan, J. E. Ness, A. Villalobos, A. Gurney, J. Minshull, C. Gustafsson, PLOS ONE 2009, 4, e7002.

[17] a) D. Summerer, A. Marx, Angew. Chem. 2002, 114, 3778; Angew. Chem. Int. Ed. 2002, 41, 3620; Angew. Chem. 2002, 114, 3778; b) K. B. M. Sauter, A. Marx, Angew. Chem. 2006, 118, 7795; Angew. Chem. Int. Ed. 2006, 45 7633; c) M. Strerath, C. Gloeckner, D. Liu, A. Schnur, A. Marx, ChemBioChem 2007, 8, 395; d) C. Gloeckner, K. B. M. Sauter, A. Marx, Angew. Chem. 2007, 119, 3175; Angew. Chem. Int. Ed. 2007, 46, 3115.

[18] R. Kranaster, A. Marx, Angew. Chem. 2009, 121, 4696; Angew. Chem. Int. Ed. 2009, 48, 4625.

[19] a) J. Haseloff, W. L. Gerlach, Nature 1988, 334, 585; b) A. Jenne, J. S. Hartig, N. Piganeau, A. Tauer, D. A. Samarsky, M. R. Green, J. Davies, M. Famulok, Nat. Biotechnol. 2001, 19, 56; c) M. Wieland, J. S. Hartig, Angew. Chem. 2006, 118, 6007; Angew. Chem. Int. Ed. 2006, 45, 5875.

[20] a) D. Rueda, J. Hsieh, J. J. Day-Storms, C. A. Fierke, N. G. Walter, Biochemistry 2005, 44, 16130; b) T. Betteridge, H. Liu, H. Gamper, S. Kirillov, B. S. Cooperman, Y. M. Hou, RNA 2007, 13, 1594.

[21] a) Y. Komine, T. Adachi, H. Inokuchi, H. Ozeki, J. Mol. Biol. 1990, 212, 579 ; b) Transfer RNA Database, University of Leipzig.

[22] a) M. S. Boosalis; J. Petruska; M. F. Goodman, J. Biol. Chem. 1987, 262, 14689; b) Creighton, L. B. Bloom, M. F. Goodman, Methods Enzymol. 1995, 262, 232.

[23] S. Obeid, M. Yulikov, G. Jeschke, A. Marx, Angew. Chem. 2008, 120, 6886; Angew. Chem. Int. Ed. 2008, 47, 6782.

[24] J. Qu, R. Morita, M. Satoh, J. Wada, F. Terakura, K. Mizoguchi, N. Ogata, T. Masuda, Chem. Eur. J. 2008, 14, 3250.

Received: July 6, 2010

Published online on August 23, 2010 